\section{rev Psi}

Revista de Psicología (UNLP)

https://revistas.unlp.edu.ar/revpsi

\title{
Percepciones sobre amor, compromiso, fidelidad y pareja en jóvenes universitarios de Quito
}

\author{
Marie-France Merlyn Sacoto ${ }^{1} \quad$ Liliana Jayo $^{1}$ \\ Rodrigo Moreta-Herrera ${ }^{1}$
}

Correspondencia

mfmerlyns@puce.edu.ec

Filiaciones institucionales

${ }^{1}$ Pontificia Universidad Católica del

Ecuador

\section{Resumen}

El objetivo de la investigación fue explorar las percepciones del amor, compromiso, fidelidad y pareja en estudiantes universitarios. En este estudio descriptivo de corte transversal, 590 jóvenes (56,4\% mujeres y 43,6\% hombres), respondieron a una encuesta de 77 ítems. Los resultados indican que en este grupo existe una versión "mixta” del amor, que combina el rechazo de la versión tradicional del amor con nociones del amor deseo, racional, romántico y posmoderno. Las nociones de compromiso, fidelidad y constituyentes de la pareja permanecen intactas, excepto en lo concerniente a la perdurabilidad del vínculo. Se encontraron diferencias por género: las posturas femeninas tienden a ser más marcadas que las masculinas, evidenciando un posicionamiento más fuerte de las mujeres en los temas analizados.

\section{Palabras clave}

percepciones | amor | compromiso | fidelidad | pareja | género

\section{Cómo citar}

Merlyn Sacoto, M.-F., Jayo, L. y MoretaHerrera, R. (2020). Percepciones sobre amor, compromiso, fidelidad y pareja Revista de Psicología, 19(2), 3-23. doi: en jóvenes universitarios de Quito. $10.24215 / 2422572$ XE063

Recibido

22 ene. 2020

Aceptado

25 jul. 2020

Publicado

14 sep.. 2020

Editor

DOI

$10.24215 / 2422572$ XE063
Nicolás Alessandroni | Facultad de Psicología, Universidad Autónoma de Madrid (España)
ISSN

2422-572X

Licencia

(c) Copyright: Merlyn Sacoto, M..F. et al. Licencia de Cultura Libre CC-BY $\underline{4.0}$

Entidad editora

RevPsi es una publicación de la

Facultad de Psicología (Universidad

Nacional de La Plata, Argentina)

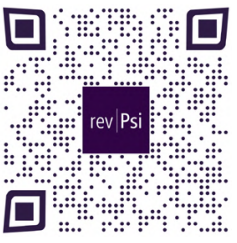

ACCESO ABIERTO DIAMANTE 


\section{Percepções sobre amor, compromisso, fidelidade e casal em universitários de Quito}

\section{Resumo}

O objetivo da pesquisa foi explorar as percepções de amor, compromisso, fidelidade e casal em estudantes universitários. Neste estudo descritivo de corte transversal, 590 jovens ( $56,4 \%$ mulheres e $43,6 \%$ homens) responderam a uma pesquisa de 77 itens. Os resultados mostram que nesse grupo há uma versão "mista" do amor, que combina a rejeição da versão tradicional do amor com noções de desejo, amor racional, romântico e pós-moderno. As noções de compromisso, fidelidade e constituintes do casal permanecem intactas, exceto no que se refere à durabilidade do vínculo. Diferenças por gênero foram observadas: as posições femininas tendem a ser mais marcantes que as masculinas, evidenciando um posicionamento mais forte das mulheres nos sujeitos analisados.

\section{Palavras-chave}

percepções | amor | compromisso | fidelidade | casal | gênero

\section{Perceptions about love, commitment, fidelity and couple in university students of Quito}

\section{Abstract}

The objective of the research was to explore the perceptions of love, commitment, fidelity and couple in university students. In this cross-sectional descriptive study, 590 young people ( $56.4 \%$ women and $43.6 \% \mathrm{men}$ ), answered a survey of 77 items. The results show that in this group there is a "mixed" version of love, which combines the rejection of the traditional version of love with notions of desire, rational, romantic and postmodern love. The notions of commitment, fidelity and constituents of the couple remain intact, except as regards to the durability of the bond. Differences by gender were observed: the feminine postures tend to be more marked than the masculine, showing a stronger positioning of women in the analyzed subjects.

\section{Keywords}

perceptions | love | commitment | fidelity | couple | gender 


\section{Aspectos destacados del trabajo}

- La versión "mixta" del amor en los jóvenes combina amor deseo, racional, romántico y posmoderno.

- La noción clásica de pareja permanece intacta (criterios de: exclusividad, permanencia, felicidad, complementariedad, visión similar).

- El compromiso en la relación es importante, implícito y es un ideal para ambos géneros.

- El binomio compromiso-fidelidad permanece intacto.

El amor es objeto de interés e investigación desde diferentes ópticas y desde hace muchos años. Las conceptualizaciones y definiciones del mismo son variadas y abarcan desde las posturas que lo visualizan como algo netamente biológico hasta aquellas más filosóficas, pasando por visiones sociales y psicológicas. Inclusive, se considera que el amor atraviesa relaciones humanas de toda índole, existiendo así diversos tipos de amor (materno-filial, de pareja, fraternal, a Dios, a la naturaleza y otros). Sin embargo, el que más atención recibe es definitivamente el amor de pareja. Incluso en este tipo de amor específicamente, existen varias perspectivas de análisis, desde las más biologistas, pasando por las históricas culturales, sociológicas y psicológicas, como se revisará a continuación.

Algunos autores consideran que el amor tiene una base biológica, lo definen como "la necesidad fisiológica de una pareja exclusiva para la cópula, la reproducción y la crianza y cuya satisfacción genera placer" (Maureira, 2011a, p. 325) y es descrito determinando las estructuras cerebrales, circuitos y neurotransmisores que sustentan diversos aspectos como el deseo sexual, el amor romántico, el apego y hasta la monogamia y la fidelidad (Conill, 2016; Maureira, 2011b). Hay que considerar que la base biológica es únicamente uno de los determinantes de las conductas humanas, que se ven influenciadas por referentes sociales y psicológicos más complejos. Por ello, existen otras definiciones más generales del amor, por ejemplo, la de los tres pilares sociales (compromiso, romance e intimidad) que se complementan con el factor biológico (Maureira, Flores, Rojas, Montiel, Valenzuela y Veganzones, 2017). Se puede entonces sostener que el amor es un fenómeno complejo, que va mucho más allá de lo biológico, siendo una experiencia a la vez subjetiva e inscrita en lo sociocultural.

Sin intención de generar la percepción de una concepción lineal del amor, en la cual un tipo de amor acaba y otro nuevo surge, se presenta a continuación la visión socio histórica del amor, que describe los ideales que se construyeron socialmente a través del tiempo, haciendo del amor un constructo determinado, sustentado y mantenido dentro del orden social. Así, a través del tiempo, aquello que se considera "amor" ha evolucionado y adquirido diferentes matices que reflejan la constitución misma de 
la sociedad que lo creó. Falavigna (2008) propone la siguiente clasificación histórica del amor: (1) amor fuerza o prehistórico, (2) amor belleza o griego, (3) amor patrimonial o romano, (4) amor cortés o medieval, (5) amor deseo o renacentista, (6) amor romántico (1700-1800), (7) amor existencialista (siglo XX) y (8) amor insignificante o posmoderno.

Si bien se considera que las concepciones de amor más antiguas han desaparecido, en la actualidad existe un cierto consenso en que los ideales y propuestas del amor romántico aún marcan la pauta de las relaciones de pareja (García, Hernández y Monter, 2019; Bonilla, Rivas y Vázquez, 2017). Esta noción de amor se generó en el romanticismo de finales del siglo XVIII y durante todo el XIX puso a las emociones en el centro de la vivencia de las parejas. La relación se convirtió en el eje de la existencia, siendo el espacio donde se buscaba la plenitud sentimental, el bienestar permanente y hasta el sentido de la vida (Sánchez-Sicilia y Cubells, 2018; Falavigna, 2008). El amor se posicionó como el eje de la relación de pareja, condición sine qua non para su existencia y se constituyó como un ideal total y magnificado, destinado a perdurar toda la vida (García, Fuentes y Sánchez, 2016), asentándose en la institución legal del matrimonio (Corona y Rodríguez, 2000). Desde esa perspectiva, el amor romántico determina lo que significa enamorarse, imponiendo creencias idealizadas en torno a este sentimiento ("mitos" del amor romántico).

Por otro lado, el amor romántico estereotipó los roles del hombre y de la mujer al presentar al género masculino como más racional y al femenino como sentimental, soñador y emocionalmente frágil (Falavigna, 2008) lo que colocó a la mujer en una postura de desigualdad y sumisión (Montenegro, 2013). Los miembros de una pareja llegaban entonces en condiciones asimétricas a la relación lo que, sumado a las expectativas generadas por los mitos del amor romántico, provocó (y sigue provocando) "la aceptación, normalización y justificación de comportamientos ofensivos y abusivos" (Montenegro, 2013, p. 12-13). Un ejemplo es el mito llamado falacia del cambio por amor, que lleva a las mujeres a soportar actitudes negativas de parejas agresivas o violentas porque creen que esta va a cambiar con el tiempo por amor.

El siglo XX llegó asociado a transformaciones profundas, sobre todo en sus últimas décadas. Factores relacionados con la revolución sexual, la economía mundial y el adelanto tecnológico cambiaron las nociones del amor. Surgió así una primera propuesta de amor, marcado por el individualismo, la igualdad de los miembros de la pareja en el dar y recibir emocional y que ponía en el núcleo de la pareja a la satisfacción sexual, garante de su continuidad (Giddens, 2010). Este tipo de amor llamado "confluente" fue claramente influenciado por la revolución sexual. Por otro lado, la globalización, masificación tecnológica y comunicación instantánea, definieron nuevas maneras de trabajar, comunicar, vivir e incluso de pensar y relacionarse. Es así que Illouz (2012) menciona que el capitalismo determina al amor, "dando lugar a lo que ella denomina un 'capitalismo emocional', en el que todo se resume en el coste-beneficio, prima la ausencia de compromiso, el desamor se convierte en algo cada vez más usual y gana terreno el 'capital sexual'" (Rodríguez-Santero, García- 
Carpintero y Porcel, 2017, p. 2). Sin llegar a anular al amor, en este contexto y pese a la pervivencia de los ideales del amor romántico, surgieron nuevas nociones de amor llamadas "posmodernas", entre ellas la del "amor líquido" (Bauman, 2005).

Así, en el siglo XXI las personas mantienen una postura dual frente a las relaciones de pareja. Por un lado, ansían los vínculos y la cercanía, pero, al mismo tiempo, temen que estos les hagan perder su individualidad y que se conviertan en cadenas que les resten libertad (Bauman, 2005). De esta manera, en la posmodernidad el sentimiento llamado "amor" se refiere a vínculos fugaces y efímeros en los que se trata de tener lazos relacionales y, a la vez, de mantenerlos débiles "para poder desanudarlos" (Sánchez-Sicilia y Cubells, 2018, p. 153). En esta situación las personas "consumen" las relaciones como lo hacen con otros productos, desechándolas cuando no cumplen con sus expectativas (Hernández, González y Regino, 2016).

La perspectiva histórica del amor antes expuesta sirve para poner al individuo en un contexto, pero se debe tomar en cuenta que aunque la sociedad provee de modelos culturales frente al amor, el sujeto se posiciona frente a estos y los resignifica: son como prototipos, ideales "que se vuelven un recurso para comparar pensamientos, sentimientos y acciones. Sirven, por ejemplo, para decidir si uno está realmente enamorado de alguien, para evaluar si uno está siendo amado por alguien o de qué manera y qué tanto" (Rodríguez Salazar y Pérez Daniel, 2007, p.176). Estamos en el terreno de las representaciones sociales, teoría que permite entender la compleja interacción entre estos modelos culturales y el individuo. Las representaciones sociales son conocimientos compartidos construidos dentro de un grupo que contribuyen a la edificación de una realidad común (Jodelet, 1986; Moscovici, 1988, Rodríguez Salazar, 2013). No por ello son estáticas o rígidas, toda vez que las personas se apropian de ellas y las particularizan en un proceso de individuación que las transforma (Valsiner, 2003, en Castorina y Barreiro, 2010). No se trata de decir que lo subjetivo es el resultado de un contexto histórico sino de entender que el sujeto puede reajustar lo que le ofrece la cultura en un momento dado, incluso haciendo suyos varios modelos al mismo tiempo, creando sus propias representaciones. Sin embargo, el contexto histórico y cultural en el que se producen las representaciones sociales debe ser entendido. En otras palabras:

(...) la idealización sobre el amor se gesta históricamente, de tal manera que se construyen imágenes o ideas que forman parte de la subjetividad de las personas (...). Estos imaginarios contienen a su vez significados, que revelan las estructuras simbólicas de una sociedad dada, y que al mismo tiempo se conjugan con los discursos, ideas, percepciones, etcétera. Lo importante es tener presente cómo se vive el sentimiento de manera particular y el significado que se le otorga al amor en cada época histórica. (García Villanueva, Hernández Ramírez y Monter Arizmendi, 2019, p. 242).

Finalmente, existen concepciones sobre el amor desde la psicología, el psicoanálisis y el humanismo, cuyas contribuciones al tema son de gran interés. Así, por ejemplo, 
la teoría de Sternberg (1986) sostiene que el amor tiene tres componentes: la pasión, que se refiere a un intenso deseo de unión y a la atracción sexual; la intimidad, que es un sentimiento de afecto, unión y cercanía con el otro; y el compromiso, que son las expectativas de mantener la relación y permanecer juntos (Almeida, 2013). En las concepciones psicoanalíticas, por otro lado, el amor es planteado conjuntamente con la noción de altruismo. En la relación con un otro significativo se juega la capacidad de experimentar gratitud por el amor recibido y el deseo de dar amor en correspondencia a ello: "esta reciprocidad muestra el altruismo presente en una relación sexual amorosa y madura" (Seelig y Rosof, 2001, p.955). Finalmente, Fromm (1959/2015) desde el humanismo, describe al amor como un arte, que requiere de disciplina para su dominio. En relación al amor de pareja específicamente, este es descrito como un anhelo de fusión con el otro, pero que va más allá del sentimiento amoroso, ya que conlleva una decisión, un juicio, una promesa: se trata de "amar desde la esencia del ser y vivenciar a la otra persona en la esencia de su ser” (p. 79). Estas tres teorizaciones tienen en común el centrarse principalmente en el fenómeno subjetivo del amor, tanto a nivel relacional (inter psíquico) como intrapsíquico.

El amor es, en todo caso a nivel de pareja, considerado muchas veces como un aspecto necesario tanto para iniciar la misma como para mantenerla (Nuñez, Cantó-Milà y Seebach, 2015). El estar en pareja trae así mismo implícitos a veces otros aspectos como la fidelidad y el compromiso con la otra persona. Ambos conceptos reciben atención en diversas investigaciones, que los definen, buscan causas y consecuencias de su presencia/ausencia en las relaciones de pareja.

La fidelidad, por un lado, se relaciona con mecanismos neurobiológicos complejos (Maureira, 2011a, 2011b). Más allá de ello, la fidelidad es definida comola exclusividad que subyace la relación de pareja. Paradójicamente, es muchas más veces estudiada debido a su ausencia (infidelidad), la que parece ser más fácil de definir. La infidelidad comprende acciones que van desde la ruptura de la exclusividad amorosa o de aspectos de intimidad emocional y/o sexual (Gonzáles, Martínez-Taboas y Martínez, 2009; Pinto, 2012; Castro, 2013), hasta aquellas más exhortantes y peyorativas como la traición y la mentira (Nuñez et al., 2015; Castro 2013). La infidelidad, como objeto de estudio, es examinada en sus posibles causas, estableciéndose incluso clasificaciones. Como causas de la infidelidad se han explorado factores biológicos (Maureira, 2011a), de personalidad, sexuales, emocionales, religiosos (en ausencia) y el aburrimiento, el distrés, la depresión, la oportunidad, etc. (Pinto, 2012; González et al., 2009). Las clasificaciones de la infidelidad usualmente hablan de dos tipos de infidelidad: la sexual y la emocional (González et al., 2009; Sirvent, 2011; Pinto, 2012). La infidelidad sexual consiste en tener actividad sexual con otra persona que no sea la pareja mientras que la emocional se refiere a la "canalización de los recursos emocionales, tales como el amor, el tiempo y la atención, hacia una persona externa a la relación" (Castro, 2013, p. 39). En los últimos años y con el avance de la tecnología, lo que se considera infidelidad es ampliado para incluir temas como "el cibersexo, el contemplar pornografía, distintos niveles de intimidad física como besos y caricias no necesariamente sexuales y cualquier involucramiento emocional 
que ocasione deterioro del vínculo amoroso oficial" (Pinto, 2012, p. 191), aunque en el tema de la virtualidad haya discrepancia de si se trata o no de infidelidad (Nuñez et al., 2015). Si la infidelidad recibe tanta atención a nivel investigativo es porque es un fenómeno bastante común o tal vez más evidente en las últimas décadas. Así, una encuesta realizada en Latinoamérica sobre los hábitos sexuales de las personas, revela que el $63 \%$ de los entrevistados han sido infieles por lo menos una vez en su vida (Tendencias Digitales, 2010).

Por otra parte, de la mano de la fidelidad viene el compromiso. Ambos conceptos son presentados incluso a veces como binomio (Sirvent, 2011). El compromiso es definido como "el interés y la responsabilidad que se siente por una pareja y por mantener dichos intereses con el transcurso del tiempo" (Yela, 1997, en Maureira, 2011a, p. 328) y las personas deben proyectarse con la pareja y consolidar la idea de permanecer junto a ella (Maureira et al., 2017). El compromiso tiene dos formas: una a corto plazo, que tiene que ver con "la decisión que se toma conscientemente de desear y querer iniciar un proyecto de vida en común con la persona amada" (Ojeda, Torres y Moreira, 2010, p.129). La otra, a largo plazo, describe al compromiso como necesario para mantener el vínculo a través del tiempo, erigiéndose muchas veces como el representante de la probabilidad de que la relación permanezca o perdure (Torres y Ojeda, 2009).

En la posmodernidad, con el auge del individualismo, las concepciones de fidelidad y compromiso se ven claramente desafiadas (Swilder, 2001, en Sirvent, 2011) y tal vez van perdiendo peso en el establecimiento de las nuevas relaciones de pareja. La misma noción de lo que es una pareja ha cambiado a través del tiempo. La tríada del amor romántico (heterosexualidad, monogamia y perdurabilidad) se sostiene con mucha dificultad en la época contemporánea, en donde emerge cada día más crítica hacia esta concepción tradicional del amor y de las relaciones de pareja (Herrera, 2016).

Actualmente, realidades como las nuevas identidades sexo genéricas abren el abanico a nuevos tipos de relaciones que van más allá de la heteronormativa, lo que quizá también da lugar a otros tipos de relaciones, llamadas "no monogamias éticas", que se proponen como nuevas opciones relacionales a nivel sexual o afectivo/sexual (swingers, relaciones “abiertas", poliamor, anarquía relacional) las cuales rompen con la absoluta monogamia que imperaba en el siglo anterior (De la Hermosa, 2018). En este sentido, por ejemplo el poliamor es entendido como una posibilidad relacional válida en la cual las personas encuentran bienestar (Pérez y Palma, 2018). Finalmente, las estadísticas muestran que la perdurabilidad del vínculo se está difuminando: en Ecuador, en una década (entre 2006 y 2016) los divorcios se incrementaron en un 83.5\% (Instituto Nacional de Estadísticas y Censos, INEC, 2017). El matrimonio, como tal, también deja de ser el estado de compromiso ideal buscado, presentando en el mismo período una baja del $22 \%$ (INEC, 2017). La relación per se ya no es un objetivo como tal y la soltería se concibe como una opción totalmente válida de vida, en la cual la persona puede sentirse realizada, opción que es acogida por un número creciente de personas (The Times, 2008, en García, Salvador y Guzmán, 
2012). Principios como perdurabilidad, exclusividad, complementariedad, que sustentaban la idea de una pareja, se tambalean en este panorama.

Numerosas investigaciones muestran que en el contexto actual, se encuentra versiones particulares respecto la pareja, el amor la intimidad, el compromiso y otros aspectos, en los diferentes grupos de jóvenes estudiados (Sirvent, 2011; BlandónHincapié y López-Serna, 2016; Rocha, Avendaño, Barrios y Polo, 2016; Hernández et al., 2018; Sánchez-Sicilia y Cubells, 2018). Así, Sirvent (2011) expone por ejemplo que, en la franja de edad de 17 a 29 años de las personas de su estudio, el $68 \%$ piensa que la confianza alimenta el amor, 66\% insiste en la exclusividad, 62\% le da importancia a la fidelidad. Pero por otro lado existe el abandono de la noción de que el compromiso de estar juntos y los hijos alimenta al amor (solamente el 19\% están de acuerdo) o que la pareja es la forma ideal de convivencia (51,4\%), llama la atención también que se abandona el mito o idea de la predestinación de una sola persona en 59,5\% de los jóvenes. Por su parte, Hernández et al. (2016) concluyen que existe una "persistencia de elementos románticos que conviven con otros propios del amor posromántico" (p. 1488). Rocha et al. (2016) encuentran que el amor ideal se caracteriza por "un amor basado en el compromiso, la pasión, atracción física, intimidad y finalmente el sacrificio" (p. 174). En otra muestra, Blandón-Hincapié y López-Serna (2016) concluyen en su investigación que persiste en los jóvenes la búsqueda de relaciones sólidas y estables pese al contexto de ligereza y de relaciones de pareja atravesadas por la incertidumbre, la transitoriedad y la individualidad. Por otro lado, realizando un análisis cualitativo del discurso de un grupo de adolescentes Sánchez-Sicilia y Cubells (2018), encuentran también la coexistencia de nociones del amor romántico con las del amor líquido, e incluso la presencia de argumentos pragmáticos que denotan un tipo de pensamiento más racional que convive con la irracionalidad de lo emocional-romántico. Finalmente, Rodríguez-Santero et al. (2017) encuentran que los jóvenes rechazan las relaciones esporádicas y sin implicación emocional, manteniendo un concepto idealizado y romántico del amor, manifestando que el amor es renuncia, negación del individualismo, entrega y sacrificio. Todas estas investigaciones nos muestran que cada grupo social estudiado se apropia a su manera de los temas relacionados con la pareja y produce una representación nueva y particular al grupo, lo que impide generalizaciones en el tema.

Dentro de este panorama tan complejo, se debe considerar adicionalmente que la realidad de los dos miembros de una pareja puede ser diferente, estando atravesada por factores como el género. Así, algunos estudios en temas relacionados encuentran diferencias en las percepciones de varones y mujeres en cuanto a: los estilos de amor (Rodríguez-Santero, García-Carpintero y Porcel, 2017), la percepción social de la infidelidad (Espinoza, Correa, García y Barragán, 2014), la preferencia de estilos de amor (Ferrer, Bosh, Navarro, Ramis y García, 2008), las estrategias a la hora de involucrarse en una relación sexual a corto plazo -ligar- o a largo plazo -relación amorosa- (Yela, 2012), entre otras.

Los cambios descritos en el contexto social, unidos a las cifras que presentan las 
estadísticas, hacen necesario plantearse la pregunta sobre la situación actual de estos temas en la sociedad ecuatoriana: ¿han cambiado en los jóvenes ecuatorianos las percepciones sobre el amor, la fidelidad, el compromiso y la pareja? Para responder a esta pregunta, que es el eje del presente estudio, se realizó un estudio exploratorio descriptivo transversal sobre el amor, el compromiso, la fidelidad/infidelidad y otros temas que constituyen los componentes de una pareja, en una muestra de jóvenes universitarios ecuatorianos. Asimismo, vista la diferencia encontrada sobre la influencia del género en investigaciones sobre temas similares, cabe la pregunta de que si esta se presentará también en los temas investigados. Este factor ha sido considerado en los análisis.

\section{Metodología}

\section{Diseño}

El presente estudio es un diseño de tipo descriptivo, comparativo, de muestras independientes por género y de corte transversal en el que se quiere conocer las diferencias existentes por grupos sobre la concepción del amor, el compromiso, la fidelidad y la pareja en jóvenes universitarios.

\section{Participantes}

Participaron en el presente estudio 590 jóvenes de una institución de educación superior de la ciudad de Quito, 56,4\% mujeres y 43,6\% hombres. La muestra es representativa de la población de esta institución que cuenta con 10.532 estudiantes de grado y en la cual el $57 \%$ son mujeres y el $43 \%$ hombres. En cuanto a la edad, el promedio de los hombres de la muestra es de 21.13 años (D.T.=1.99) y el de las mujeres es de 20.69 años (D.T.= 2.03). La edad mínima de toda la muestra es de 18 años y la máxima de 25. Los estudiantes pertenecían a las todas las facultades de la institución. La selección de los participantes se realizó a través de un muestreo probabilístico por voluntariado. Participaron todos aquellos estudiantes que fueron informados del proyecto y que llenaron la encuesta en línea mientras estuvo activa (entre noviembre de 2018 y marzo del 2019).

\section{Instrumento}

A partir de un estudio amplio realizado dentro de la institución sobre el tema "Sexualidad en la Juventud" con estudiantes de 18 a 25 años, usando la metodología de grupos focales ( $\mathrm{n}=120$ hombres y $\mathrm{n}=122$ mujeres) se detectó la necesidad de recabar información sobre las percepciones del amor, el compromiso, la fidelidad y la pareja de los jóvenes. Se decidió hacerlo a través de una encuesta ad-hoc, elaborada en torno a los temas que surgieron en los grupos focales y a partir de la revisión de la literatura pertinente. Para las temáticas en cuestión se desarrollaron originalmente 90 ítems, que fueron sometidos a validación por 3 expertos psicólogos; para la versión final solo se tomaron en cuenta aquellos ítems en los que se obtuvo al menos 
2 criterios de acuerdo; la versión final de la encuesta constó de 77 ítems y se enfocó en los siguientes aspectos: (a) concepciones sobre el amor (37 ítems), (b) compromiso (10), (c) fidelidad/infidelidad (24) y (d) condiciones para la pareja (6). Se ejecutó un estudio piloto con estudiantes para realizar adaptaciones y adecuaciones necesarias a los ítems, sobre todo en el tema de claridad de redacción de los ítems.

En cuanto al amor, las percepciones fueron exploradas a través de 37 ítems construidos alrededor de la clasificación del amor de Falavigna (2008). El manejo de nociones socio históricas del amor para la presente investigación es una elección de los autores, que no excluye que existan visiones diferentes, más complejas y centradas en las personas y su experiencia subjetiva, que tengan hallazgos válidos sobre el tema. Existe una multiplicidad de abordajes sobre el amor, y creemos que cada investigación empírica con sus particularidades contribuye a ver el fenómeno con una perspectiva complementaria. Se excluyó de las nociones de Falavinga (2008) al amor fuerza o prehistórico, amor belleza o griego, al amor cortés, por considerárselos no vigentes actualmente ya que los jóvenes no hicieron alusión a ellos en los grupos focales. Se tomaron en cuenta: el amor tradición, amor posmoderno y al amor romántico. En la redacción de los ítems de este último, adicionalmente se usó la clasificación de los mitos en cuatro grupos propuesta por Montenegro (2013).

Con base en la revisión teórica, se construyeron los ítems sobre compromiso y fidelidad/infidelidad. El compromiso, en su versión positiva, fue descrito en 4 ítems positivamente (duración, importancia, caracterizar a la relación y aspiración de ambos géneros) y en 6 ítems de manera negativa (anacrónico, peligroso, limitante, aspiración únicamente femenina, temor masculino y convención social). Para explorar la fidelidad/ infidelidad, se construyeron 24 ítems, divididos en tres subtemas: razones para la fidelidad, para la infidelidad y conductas de infidelidad. Los factores de protección para la infidelidad fueron llamamos "razones para la fidelidad" (8 ítems). Las causas de la infidelidad se retomaron en 10 ítems. Finalmente, en 6 ítems se exploraron las conductas de infidelidad sexual (tener relaciones sexuales), de contacto (besarse), sexual ampliada (posesión de pornografía), romántica (flirteo en persona o en chat), íntima (compartir cosas personales) y virtual (aplicaciones para citas de parejas).

Para las respuestas de estos ítems se utilizó una escala de tipo Likert de cinco opciones que va desde totalmente en desacuerdo (1) a totalmente de acuerdo (5).

Losíndices de confiabilidad son adecuados para la escala de Amor $(\alpha=.80)$ y Fidelidad/ infidelidad $(\alpha=.80)$. La fiabilidad para la escala de Compromiso es admisible $(\alpha=.60)$, ya que se considera que valores de alfa de 0.5 o 0.6 son aceptables en las fases exploratorias de una investigación (Jisu, Delorme y Reid, 2006); posiblemente el bajo número de ítems explica este valor (Vaske, Beaman y Sponarski, 2017). También se analiza la adecuación muestral para la conformación de factores según el modelo propuesto. Para ello KMO $=0.85$ indica que es adecuada. Así mismo, se contrasta si el modelo factorial es adecuado por medio de la prueba de esfericidad de Barttlet (5381.64, $\mathrm{p}=.000)$, en el cual se concluye que sí lo es. 
En cambio, para explorar el tema de pareja se propusieron 6 ítems en los cuales los participantes debían contestar "sí" o "no" a las condiciones que creían que debe tener una pareja. Las condiciones propuestas fueron: (1) exclusividad, (2) permanencia en el tiempo, (3) presunción de felicidad, (4) complementariedad, (5) visión similar de la vida y (6) experiencias similares.

\section{Procedimiento}

La encuesta fue aplicada en línea. Si bien algunos estudios cuestionan los aspectos negativos de las evaluaciones en línea (Steger, Schroeders, y Gnambs, 2020), se consideró que en este caso era el tipo de aplicación óptima por la facilidad de acceso a la población y la tranquilidad requerida por los participantes para contestar a las preguntas en relación al alto número de ítems. Se consideró que se obtendrían respuestas confiables por las siguientes razones: (1) no se trataba de una evaluación de habilidades específicas, sino de actitudes personales frente a los temas, (2) la participación era enteramente voluntaria, lo que determinaba que el participante podía no finalizar su encuesta si así lo deseaba, y (3) los participantes no obtenían ninguna retribución o sanción por realizarla o no. Todos los estudiantes de grado fueron contactados a través de un mensaje de correo electrónico en donde se explicaba el objetivo de la encuesta, su duración aproximada y los riesgos y beneficios de su participación. Se proveía en el mismo un vínculo para que accedieran a la encuesta. En esta constaba el consentimiento informado aprobado por el Comité de Ética para ratificar la voluntad de participar en la investigación. La encuesta permaneció abierta en la internet durante 2 meses para ser completada por cuantos estudiantes lo quisieran. Después de este tiempo se hizo una revisión de la información recolectada y se constató que existía una desproporción en las respuestas por género. Así, existían más respuestas de mujeres que varones. Se decidió mantener la encuesta abierta por 2 meses más y se implementaron estrategias para incrementar la participación masculina. Entre ellas: correos electrónicos solo a los estudiantes varones de la institución y hablar directamente en clase solicitando participación de varones. De esta manera, se consiguió equilibrar la muestra en cuanto a género y que fuera representativa de la población. Una vez recolectada la información, se procedió al análisis de datos utilizando el programa SPSS versión 25 (IBM Corp, 2017).

\section{Resultados}

Análisis descriptivo de las medias por dimensión. Para poder tener una idea de la tendencia en el acuerdo/desacuerdo de los participantes con las afirmaciones propuestas sobre los diferentes temas, se realizó un análisis descriptivo de las medias en función de su ubicación en relación a la escala Likert.

\section{Componente de amor}

Los resultados expuestos en la Tabla 1 muestran que son pocas las percepciones con la que los participantes están medianamente de acuerdo: disolución de la 
unión (formulado inversamente en amor tradición), atracción física y presencia de placer (amor deseo), libertad y madurez y elección racional (amor existencialista). Dentro del amor romántico, de los 19 ítems propuestos, solo en cuatro la media indica acuerdo: cambio por amor, omnipotencia del amor, discusiones (únicamente en varones) y renuncia a la intimidad. En cuanto al amor posmoderno, de los 7 ítems solo en dos hay acuerdo: dificultad de la estabilidad e individualismo. En todos los demás ítems de las diferentes propuestas de amor las medias indican que existe desacuerdo o total desacuerdo según la percepción de los participantes.

\section{Componente de compromiso}

Los puntajes de las medias en Tabla 2 muestran que los participantes obtienen puntajes más altos en la mayoría de ítems que describen al compromiso de manera positiva (importancia, propio de la relación y compromiso compartido), salvo por el ítem de duración donde la tendencia va más hacia el desacuerdo (Tabla 3). Por otra parte, los puntajes de las medias de la mayoría de los ítems que describen negativamente al compromiso tienden a ser bajos (anacronismo, peligro, limitación, aspiración femenina y temor masculino), salvo por el puntaje de la media en convención social, que se observa un poco más alto. Globalmente, los jóvenes de la muestra valoran entonces de una manera positiva al compromiso, salvo en lo relativo a la duración y a la convención social.

\section{Componente de fidelidad/ infidelidad}

Los factores que obtuvieron valores más altos que explican la fidelidad fueron las creencias religiosas, convencimiento personal y tranquilidad personal las cuales son razones endógenas, así como el compromiso con la pareja. Mientras que las razones externas o más superfluas son rechazadas (costumbre, control social, miedo a enfermedades, factores económicos) (Tabla 3$)$.

En cuanto a la infidelidad, de todas las causas propuestas los participantes únicamente manifiestan desacuerdo con la presión social y la moda, hallando entonces varias razones detrás de las conductas de infidelidad, siendo la que obtiene mayor acuerdo la insatisfacción emocional. Finalmente, en cuanto a las conductas propuestas como posiblemente infieles, se encontró que únicamente el chatear con una persona sobre asuntos personales no es considerado infidelidad. En todas las demás conductas hay un acuerdo, siendo las de besarse o tener relaciones sexuales con otra persona en las que se observan los puntajes de acuerdo más altos.

\section{Componente de condiciones para que exista una pareja}

$\mathrm{Al}$ parecer, todas las condiciones propuestas son valoradas como necesarias por ambos grupos (Tabla 4). Aquellas condiciones que reciben aprobación en $60 \%$ o más de los participantes son en su orden: (1) la complementariedad, (2) la permanencia en el tiempo, (3) exclusividad, (4) presunción de felicidad (5) visión similar de la vida. Sin embargo, la condición "experiencias similares" tiene porcentajes muy parecidos a favor y en contra. 
Tabla 1. Resultados descriptivos del componente de amor

\begin{tabular}{|c|c|c|c|c|}
\hline \multirow{2}{*}{ Tema } & \multicolumn{2}{|c|}{ Hombres } & \multicolumn{2}{|c|}{ Mujeres } \\
\hline & Media & Desv. & Media & Desv. \\
\hline \multicolumn{5}{|l|}{ Amor ligado a la tradición } \\
\hline Tradición necesaria & 2.16 & 1.13 & 1.93 & 1.09 \\
\hline Amparo por unión & 2.22 & 1.13 & 2.06 & 1.15 \\
\hline Disolución de la unión & 3.24 & 1.23 & 3.71 & 1.18 \\
\hline Permanencia por hijos & 1.97 & 1.08 & 1.41 & .78 \\
\hline \multicolumn{5}{|l|}{ Amor deseo } \\
\hline Atracción física & 3.41 & 1.08 & 3.38 & 1.14 \\
\hline Presencia de placer & 3.28 & 1.14 & 3.07 & 1.29 \\
\hline Necesidad sexual & 2.91 & 1.09 & 2.84 & 1.17 \\
\hline Equivalencia sexo y amor & 1.75 & .94 & 1.47 & .79 \\
\hline Sexo únicamente & 1.90 & 1.02 & 1.44 & .79 \\
\hline \multicolumn{5}{|l|}{ Amor racional/existencialista } \\
\hline Libertad y madurez & 4,06 & 1,09 & 4.57 & .78 \\
\hline Elección racional & 3.38 & 1.22 & 3.65 & 1.16 \\
\hline \multicolumn{5}{|l|}{ Amor romántico - Grupo 1} \\
\hline Cambio por amor & 3.65 & 1.06 & 3.58 & 1.13 \\
\hline Omnipotencia del amor & 3.37 & 1.10 & 3.31 & 1.21 \\
\hline Atracción de los opuestos & 2.88 & 1.03 & 2.80 & .90 \\
\hline Sufrimiento & 1.98 & .93 & 1.35 & .62 \\
\hline Maltrato psicológico & 1.93 & 1.02 & 1.33 & .71 \\
\hline Maltrato verbal & 2.35 & 1.05 & 1.91 & .98 \\
\hline Discusiones & 3.18 & 1.18 & 2.72 & 1.23 \\
\hline Perdón absoluto & 2.20 & 1.12 & 1.8 & .95 \\
\hline \multicolumn{5}{|l|}{ Amor romántico - Grupo 2} \\
\hline Amor verdadero & 2.13 & 1.08 & 1.89 & 1.03 \\
\hline Perdurabilidad & 2.94 & 1.28 & 2.83 & 1.35 \\
\hline Media naranja & 2.58 & 1.17 & 2.17 & 1.18 \\
\hline Complemento total & 2.95 & 1.14 & 2.67 & 1.23 \\
\hline \multicolumn{5}{|l|}{ Amor romántico - Grupo 3} \\
\hline Renuncia a la intimidad & 3.20 & 1.20 & 3.12 & 1.29 \\
\hline Entrega intimidad sexual & 2.30 & 1.03 & 1.88 & .99 \\
\hline Sacrificio & 2.71 & 1.26 & 2.36 & 1.28 \\
\hline Despersonalización & 2.45 & 1.20 & 1.93 & 1.08 \\
\hline \multicolumn{5}{|l|}{ Amor romántico - Grupo 4} \\
\hline Formalización de la relación & 2.21 & 1.17 & 1.96 & 1.11 \\
\hline Unión perpetua & 2.64 & 1.28 & 2.31 & 1.34 \\
\hline Celos & 2.11 & 1.09 & 1.67 & .90 \\
\hline \multicolumn{5}{|l|}{ Amor posmoderno } \\
\hline Vínculos efímeros 1 & 2.11 & .99 & 1.98 & 1.13 \\
\hline Vínculos efímeros 2 & 2.70 & 1.18 & 2.97 & 1.31 \\
\hline Dificultad estabilidad & 3.33 & 1.28 & 3.35 & 1.38 \\
\hline No exclusividad & 2.86 & 1.23 & 2.63 & 1.30 \\
\hline Escape a la soledad & 2.01 & 1.03 & 1.55 & .93 \\
\hline Individualismo & 3.96 & 1.11 & 4.45 & .85 \\
\hline Inexistencia del amor & 2.69 & 1.19 & 2.28 & 1.07 \\
\hline
\end{tabular}

Nota: Hombres $=257$ casos; Mujeres $=333$ casos 
Tabla 2. Resultados descriptivos del componente de compromiso

\begin{tabular}{lrrrr}
\hline \multirow{2}{*}{ Tema } & \multicolumn{2}{c}{ Hombres } & \multicolumn{2}{c}{ Mujeres } \\
\cline { 2 - 5 } & Media & Desv. & Media & Desv. \\
\hline Visión positiva del compromiso & & & & \\
Duración & 2.60 & 1.18 & 2.28 & 1.18 \\
Importancia & 3.87 & 1.04 & 3.90 & 1.06 \\
Característica de la relación & 3.48 & 1.08 & 3.29 & 1.19 \\
Aspiración de ambos géneros & 3.58 & 1.04 & 3.66 & 1.04 \\
Visión negativa del compromiso & & & & \\
Anacronismo & & & & \\
Peligro & 2.20 & .94 & 1.86 & .87 \\
Limitación & 2.18 & 1.05 & 1.96 & 1.09 \\
Aspiración femenina & 2.37 & 1.16 & 1.98 & 1.11 \\
Temor masculino & 1.89 & .94 & 1.47 & .73 \\
Convención social & 2.33 & 1.07 & 2.45 & 1.08 \\
\hline
\end{tabular}

Nota: Hombres $=257$ casos Mujeres $=333$ casos

Tabla 3. Resultados descriptivos del componente de fidelidad/infidelidad

\begin{tabular}{lrrrr}
\hline \multirow{2}{*}{\multicolumn{1}{c}{ Tema }} & \multicolumn{2}{c}{ Hombres } & \multicolumn{2}{c}{ Mujeres } \\
\cline { 2 - 5 } & Media & Desv. & Media & Desv. \\
\hline Razones para la fidelidad & & & & \\
Costumbre & 2.94 & 1.18 & 2.88 & 1.26 \\
Creencias religiosas & 3.01 & 1.20 & 3.05 & 1.26 \\
Convencimiento personal & 3.99 & .97 & 4.34 & .92 \\
Compromiso con la pareja & 4.10 & 1.01 & 4.49 & .77 \\
Control social & 2.63 & 1.19 & 2.60 & 1.24 \\
Miedo a enfermedades & 2.60 & 1.19 & 2.59 & .07 \\
Factores económicos & 2.42 & 1.13 & 2.25 & .06 \\
Tranquilidad personal & 3.09 & 1.24 & 3.40 & 1.30 \\
& & & & \\
Razones para la infidelidad & & & & \\
Aburrimiento & 3.39 & 1.10 & 3.17 & 1.26 \\
Miedo al compromiso & 3.10 & 1.18 & 3.35 & 1.17 \\
Curiosidad sexual & 3.57 & 1.05 & 3.47 & 1.14 \\
Insatisfacción sexual & 3.54 & 1.03 & 3.40 & 1.28 \\
Venganza por infidelidad & 3.51 & 1.02 & 3.50 & 1.12 \\
Insatisfacción emocional & 3.64 & 1.03 & 3.76 & 1.02 \\
Personalidad & 3.09 & 1.24 & 2.97 & 1.33 \\
Interés & 3.24 & 1.12 & 3.15 & 1.15 \\
Presión social & 2.78 & 1.19 & 2.71 & 1.23 \\
Moda & 2.37 & 1.14 & 2.33 & 1.20 \\
Conductas/infidelidad & & & & \\
Besarse & 3.95 & 1.07 & 4.34 & 0.89 \\
Tener relaciones sexuales & 4.28 & .94 & 4.64 & .72 \\
Tener vídeos o imágenes sexuales & 3.29 & 1.28 & 3.57 & 1.19 \\
Chatear con otra persona & 2.47 & 1.17 & 2.60 & 1.18 \\
Coquetear / flirtear con una persona & 3.46 & 1.11 & 3.86 & 1.08 \\
Aplicaciones de ligue & 3.28 & 1.14 & 3.68 & 1.16 \\
\hline & & & &
\end{tabular}

Nota: Hombres $=257$ casos; Mujeres $=333$ casos 


\begin{tabular}{lrrrr}
\hline \multirow{2}{*}{ Tabla 4. Resultados descriptivos de las condiciones de una pareja } \\
\hline \multirow{2}{*}{ Tema } & \multicolumn{2}{c}{ Hombres } & \multicolumn{2}{c}{ Mujeres } \\
\cline { 2 - 5 } & \multicolumn{1}{c}{ Sí (\%) } & No (\%) & Sí (\%) & No (\%) \\
\hline Exclusividad & 70 & 30 & 70.9 & 29.1 \\
Permanencia en el tiempo & 76.3 & 23.7 & 69.1 & 30.9 \\
Presunción de felicidad & 69.6 & 30.4 & 59.5 & 40.5 \\
Complementariedad & 88.7 & 11.3 & 90.7 & 9.3 \\
Visión similar de la vida & 59.1 & 40.9 & 59.8 & 40.2 \\
Experiencias similares & 53.7 & 46.3 & 47.1 & 52.9 \\
\hline
\end{tabular}

Nota: Hombres $=257$ casos; Mujeres $=333$ casos

\section{Análisis comparativo por género}

Los resultados expuestos en la Tabla 5 muestran que para casi todas las nociones de amor existen diferencias significativas $(\mathrm{p}<, 05)$ de medias entre géneros: únicamente en la noción de amor posmoderno las respuestas no difieren. La tendencia de las diferencias de medias (con tamaños del efecto pequeños $[g>, 2]$ y moderados $[g>, 5]$ ) es a que las mujeres presenten medias menores que los hombres, lo que las acerca más a estar en desacuerdo con todas las nociones en lo relativo al amor tradición, deseo y amor romántico. Las diferencias más fuertes se observan en lo relativo al amor romántico (tamaño del efecto g> 6). En cuanto a la noción de amor racional/existencialista, las mujeres muestran estar más de acuerdo con las propuestas que lo definían.

En lo relativo al compromiso, se puede observar también que en la dimensión general existen diferencias significativas, seguramente generadas por las diferencias en el componente del compromiso negativo (con tamaños del efecto pequeños [g> 4]). Finalmente en la noción de fidelidad/infidelidad hay diferencias de medias significativas entre ambos grupos, generada sobre todo por la diferencia observada en el tema de las conductas de infidelidad, aunque el tamaño del efecto sea bajo ( $\mathrm{g}>$,4) (Tabla 5).

En general se observa que en las dimensiones en los que hay efecto de género se refleja que las posturas femeninas tienden a ser más radicales que las masculinas: en los componentes en los que la media tiende al acuerdo para ambos grupos, las mujeres presentan puntajes mayores de acuerdo y en los componentes en los que la media va hacia el desacuerdo en ambos grupos, el desacuerdo es mayor por parte de las mujeres.

\section{Discusión}

En este grupo de jóvenes universitarios se encontró una versión "mixta" del amor, que combina el rechazo de la versión tradicional del amor con ciertas nociones del amor deseo y con las de un amor más racional, así como con unas pocas nociones del amor romántico y algunas descripciones del amor posmoderno. Versiones mixtas del amor, con similitudes y diferencias con la encontrada en este estudio, han sido encontradas en otras investigaciones con poblaciones similares (Sirvent, 2011; Blandón-Hincapié y López-Serna, 2016; Rocha, Avendaño, Barrios y Polo, 2016; Hernández et al., 2018; Sánchez-Sicilia y Cubells, 2018). 
Tabla 5. Resultados comparativos por género en las nociones de amor, compromiso y fidelidad/infidelidad

\begin{tabular}{|c|c|c|c|c|c|c|}
\hline \multirow{2}{*}{ Tema } & \multicolumn{2}{|c|}{ Hombres } & \multicolumn{2}{|c|}{ Mujeres } & \multirow{2}{*}{$t ; p$} & \multirow{2}{*}{$g$} \\
\hline & Media & Desv. & Media & Desv. & & \\
\hline Amor ligado a la tradición & 2,40 & 0,73 & 2,28 & 0,57 & 2,$23 ; .027$ & 0.2 \\
\hline Amor deseo & 2,65 & 0,58 & 2,44 & 0,57 & 4,$43 ; .000$ & 0.4 \\
\hline Amor racional/existencialista & 3,72 & 0,97 & 4,11 & 0,79 & $-5,32 ; .000$ & 0.5 \\
\hline Amor romántico & 2,62 & 0,54 & 2,30 & 0,55 & 7,$13 ; .000$ & 0.6 \\
\hline Grupo 1 & 2,69 & 0,56 & 2,35 & 0,54 & 7,$46 ; .000$ & 0.6 \\
\hline Grupo 2 & 2,65 & 0,79 & 2,39 & 0,84 & 3,$81 ; .000$ & 0.3 \\
\hline Grupo 3 & 2,66 & 0,69 & 2,32 & 0,71 & 5,$84 ; .000$ & 0.5 \\
\hline Grupo 4 & 2,32 & 0,88 & 1,98 & 0,84 & 4,$82 ; .000$ & 0.4 \\
\hline Amos posmoderno & 2,81 & 0,57 & 2,75 & 0,57 & 1,$34 ; .182$ & - \\
\hline Compromiso & 2,71 & 0,49 & 2,53 & 0,45 & 4,$68 ; .000$ & 0.4 \\
\hline Compromiso positivo & 3,38 & 0,79 & 3,28 & 0,74 & 1,$53 ; .126$ & - \\
\hline Compromiso negativo & 2,27 & 0,72 & 2,03 & 0,65 & 4,$19 ; .000$ & 0.4 \\
\hline Fidelidad/infidelidad & 3,24 & 0,51 & 3,34 & 0,46 & $-2,48 ; .013$ & 0.2 \\
\hline Razones fidelidad & 3,10 & 0,68 & 3,20 & 0,64 & $-1,89 ; .059$ & - \\
\hline Razones infidelidad & 3,22 & 0,72 & 3,18 & 0,74 & 0,$68 ; .500$ & - \\
\hline Conductas infidelidad & 3,46 & 0,81 & 3,78 & 0,73 & $-5,18 ; .000$ & 0.4 \\
\hline
\end{tabular}

257 casos; Mujeres $=333$ casos; $g l=588$; $t$ Prueba $t$ de Student; $p$ : Significancia; $g$ : Prueba de Hedges ajustada

Los resultados muestran primeramente un desacuerdo con las propuestas tradicionales sobre el amor, que lo asentaban en la legalidad y en los hijos. Al contrario, el concepto del amor como sentimiento cuenta con un amplio acuerdo como cimiento de la relación de pareja y que causaría su disolución si no existe. Para este grupo de jóvenes, el amor se centra en la atracción física como punto de arranque y aunque el placer en las relaciones sexuales garantiza la permanencia en la pareja, rechazan las ideas de que el amor vaya a la par con la necesidad sexual o que se pueda asimilar amor con sexo. Además, en su concepción del amor, hacen suyas nociones del amor racional, como por ejemplo que exista racionalidad en la elección y libertad y madurez en la relación.

Por su parte, se puede observar que entre los conceptos propuestos los del amor romántico, en menor medida, siguen siendo un referente para los jóvenes. Aunque "el verdadero amor" no "perdona todo", sin embargo, sigue existiendo en el imaginario la falacia del cambio por amor y el mito de que si las personas se quieren todo puede solucionarse, así como la idea de que en la relación no deben existir secretos, únicas propuestas del amor romántico en las que ambos géneros están aún de acuerdo. Por un lado, puede tratarse de concepciones "residuales" que permanecen internalizadas en ellos. Por otro lado, puede estar contribuyendo al mantenimiento de estos mitos la presencia de los mismos en los productos culturales como canciones, películas, relatos etc. Cabe destacar el hecho de que los mitos del amor romántico que sustentan el maltrato (como la compatibilidad del amor con el sufrimiento, maltrato verbal y psicológico) son prácticamente rechazados aunque los varones permanezcan de 
acuerdo con la normalidad de la presencia de conflictos y discusiones. Así, es claro el efecto de género en las afirmaciones sobre la compatibilidad del amor con el maltrato y en la normalización del conflicto, que refleja seguramente un cambio más profundo en la visión de las mujeres frente a la violencia de género, fenómeno que recibe cada día más y más atención. En este cambio, seguramente impacta el hecho de que la violencia doméstica, el maltrato ligado a las relaciones de pareja y los femicidios son claramente condenados por el colectivo social en Ecuador. Actualmente existen muchas campañas en redes sociales y diversos medios sobre lo peligroso que es asociar al amor con conductas violentas que no son manifestaciones del mismo y esta presión social condujo recientemente a la aprobación de la Ley Orgánica para la Prevención y Erradicación de la Violencia de Género contra las Mujeres (2018).

En general, lo que se encuentra en nuestra investigación es que las posturas femeninas tienden a ser más marcadas que las masculinas, evidenciando un posicionamiento más fuerte en los temas analizados (amor, fidelidad, compromiso y pareja), lo que a su vez puede ser en sí mismo un efecto de la pertenencia al género, puesto que estos temas se consideran de competencia femenina en una sociedad claramente influenciada por conceptos de feminidad/masculinidad tradicionales, que definen al hombre como lógico y racional y ubican en la esfera de lo emocional y relacional a las mujeres (Cabral y García, 2000).

Volviendo al tema del amor, las ideas del amor posmoderno tampoco satisfacen del todo a los jóvenes del grupo estudiado. Si bien el amor debe centrarse primero en ellos (individualismo) y aunque reconocen que es más difícil mantener una relación en esta época, rechazan las propuestas posmodernas de la futilidad de los vínculos, del amor propugnado por el miedo a soledad, de que el amor no existe o que se sustenta solo en lo sexual, e incluso la del poliamor.

El que los jóvenes manifiesten que es más difícil mantener una relación de pareja no parece tener relación con su noción de pareja, puesto que criterios como la exclusividad, permanencia en el tiempo, presunción de felicidad en pareja, complementariedad y visión similar de la vida siguen siendo importantes para ellos. Asimismo, sus visiones sobre el compromiso tienden a ser positivas, manteniendo la idea de que este es importante, que está implícito en una relación y que es un ideal para ambos géneros. El binomio compromiso- fidelidad, permanece intacto. Los jóvenes asocian la fidelidad con valores importantes e internos de la persona como el compromiso, el convencimiento, la tranquilidad personal y las creencias religiosas, antes de atribuirlo a causas exógenas. Igualmente, no atribuyen la infidelidad a causas externas como la presión social o la moda, responsabilizando al individuo de su conducta.

Por otra parte y en concordancia con lo anterior, los jóvenes tienen una noción amplia de la infidelidad, que incluye conductas más allá de la infidelidad sexual o de contacto físico, como el coqueteo o flirteo e incluso cosas ligadas a lo virtual (posesión de imágenes sexuales y aplicaciones de ligue) excluyendo únicamente al chatear sobre asuntos íntimos. Pareciera entonces que no existe una ruptura ni un rechazo las nociones tradicionales del compromiso y la fidelidad, ni a los constituyentes de la 
pareja. El impacto se sitúa únicamente en la perdurabilidad del vínculo.

Esta coexistencia de varias versiones del amor (sobre todo del romántico con el posmoderno) y la preservación de conceptos relacionales clásicos (compromiso, fidelidad, exclusividad) se encuentra en otros estudios realizados con poblaciones similares, lo que corrobora lo expuesto en la revisión de la literatura (BlandónHincapié y López-Serna, 2016; Hernández et al., 2016; Rocha et al., 2016; RodríguezSantero et al.; 2017; Sánchez-Sicilia y Cubells, 2018; Sirvent, 2011). Los estudios de Hernández et al. (2016), y Rocha et al. (2016), por ejemplo, llegan a conclusiones similares a las del presente estudio, sobre la existencia de representaciones que combinan el amor romántico y el posmodernismo, pero se diferencian al mantener los jóvenes de sus grupos al sacrificio dentro de la noción de amor, algo que no está presente en nuestra muestra.

Resulta evidente que en todos estos estudios, incluyendo el presente, lo que se encuentra es la coexistencia de varias nociones del amor en poblaciones jóvenes. Por las diferencias encontradas en los distintos estudios, parecería que cada grupo social implicado está realizando su propia "mezcla", guardando del amor romántico aquello que parece aún positivo (y esto depende de cada contexto) y tratando de encajarlo con las versiones posmodernas del amor. Los hallazgos corroboran lo planteado por la teoría de las representaciones sociales. En la actualidad perviven las nociones del amor romántico y las posmodernas, que nutren la visión de lo que circula en una pareja; estas nociones a su vez se particularizan en los individuos, generando representaciones propias a este grupo. Esto tiene implicaciones claras para la intervención que se hace con jóvenes a nivel relacional. No se puede asumir, por ejemplo, que encontrándose en una sociedad posmoderna los jóvenes asumirán ipso facto las nociones del amor líquido.

En nuestra investigación hallamos, por el contrario, que los jóvenes rechazan muchas nociones del amor líquido, y no se autodentifican con ideas como la fugacidad de los vínculos, el temor a la soledad o la inexistencia del amor. Metodológicamente hablando, puede ser una de las limitaciones de nuestro estudio el haber solicitado que se responda a estas afirmaciones que son "socialmente negativas". Metodologías como la observación directa o el análisis del discurso resultarían más apropiadas para evidenciar la asimilación de estas nociones del amor posmoderno, como en el caso de la investigación de Sánchez-Sicilia y Cubells (2018). Ciertas afirmaciones menos duras, como la del centrar el amor en sí mismo antes de que en los demás (individualismo) o la constatación de la dificultad de mantener las relaciones a largo plazo, seguramente son más fáciles de asumir y por eso presentan aceptación en nuestro estudio.

Debemos reflexionar respecto a otro límite de nuestra investigación en este tema y es el de no haber propuesto a los participantes afirmaciones en concordancia con la definición del amor "confluente" de Giddens (2010, citado en Hernández et al., 2016), versión del amor anclada en las relaciones libres, sin ataduras, de igualdad entre los sexos. No se lo hizo por cuanto los jóvenes en los grupos focales no toparon 
el tema, pero debió de incluírselo en base a la revisión de la literatura. En este tipo de amor, el centro es el goce y disfrute de lo sexual, plasmado en una "relación pura", donde la perdurabilidad es garantizada mientras se mantenga la satisfacción entre los implicados. En el estudio de Hernández et al. (2018) el amor posmoderno fue definido de esta manera y se observa un acuerdo de los participantes con valores como "confianza, reciprocidad, cuidado y respeto, como significantes de la relación de pareja” (p. 1488). En futuras investigaciones se deberá tomar en cuenta este tema.

Los resultados obtenidos en la presente investigación muestran claramente la importancia de contextualizar los temas en la población específica, ya que esto puede dar luces a los profesionales del medio sobre el abordaje en temas de pareja en la actualidad. Así, es importante conocer que las nociones en las que se enmarcan las relaciones de pareja (amor, compromiso, fidelidad y otras) están transformándose en la actualidad, cambiando incluso las experiencias subjetivas de las personas que se involucran en una pareja (Rihm, Sharim, Barrietos, Araya y Larraín, 2017), por lo que adquiere importancia el conocer los grupos sociales con los que se va a trabajar.

En este caso, por ejemplo, la investigación realizada con jóvenes universitarios arroja luces sobre sus percepciones y permite ver un contexto con cambios positivos (como el abandono de la mayoría de mitos del amor romántico), así como evidencia temas en los que se debe aún enfatizar. Por otro lado, sería importante abrir espacios de discusión sobre estas temáticas con jóvenes, para permitir la expresión de posibles situaciones que les generen conflicto, como el abandono de las visiones tradicionales que la sociedad aún propone y el surgimiento de nuevas concepciones con las que puedan identificarse mejor.

Entre las limitaciones del estudio se debe mencionar principalmente la relacionada con el instrumento, dado que al no existir una prueba estandarizada que explore a profundidad las áreas de nuestro interés como son las concepciones del amor, el compromiso, la fidelidad y las condiciones de pareja hemos recurrido a la elaboración de un cuestionario Ad Hoc que se formuló teóricamente y posteriormente se validó su contenido por un comité de expertos y en un pilotaje. Sin embargo, aún requiere el estudio de sus distintas propiedades psicométricas y su estandarización (los primeros resultados arrojan conclusiones positivas acerca de la fiabilidad del cuestionario en varias escalas). Por lo tanto, los resultados descriptivos expuestos son preliminares y tienen limitaciones inferenciales, por lo que deben ser tomados con cautela. A futuro se recomienda elaborar nuevos estudios similares a fin de corroborar los resultados encontrados en muestras similares, así como también explorar en las propiedades psicométricas de la prueba, principalmente validez factorial con estimación robusta, convergencia y discriminación. 


\section{Referencias}

Almeida, A. (2013). Las ideas del amor de RJ Sternberg: La teoría triangular y la teoría narrativa del amor. Familia: Revista de ciencias y orientación familiar, 46, 57-86.

Bauman, Z. (2005). Amor líquido. Acerca de la fragilidad de los vínculos humanos. FCE.

Blandón-Hincapié, A. y López-Serna, L. (2016) Comprensiones sobre la pareja en la actualidad: jóvenes en busca de estabilidad. Revista Latinoamericana de Ciencias Sociales, Niñez $y$ Juventud, 14(1), 505-517. HTTPS://DOI. ORG/10.1160o/1692715X.14134271014

Bonilla, E., Rivas, E. y Vázquez, J. (2017). Tolerancia y justificación de la violencia en relaciones de pareja adolescentes. Apuntes de Psicología, 35(1), 55-61.

Cabral, B. y García, C. (2000). Masculino/femenino ¿Y yo? Identidades de género. Mérida, 10, 1-16.

Castorina, J. A. y Barreiro, A. V. (2010). El proceso de individuación de las representaciones sociales: historia y reformulación de un problema. Interdisciplinaria, 27(1), 63-75.

Castro, M. (2013). Fidelidade e infidelidade nas relações amorosas. Universidade Católica Editora.

Conill, J. (2016). La intimidad corporal y sus bases neurobiológicas. Pensamiento, 72(273), 789-807. HTTPS://DOI.ORG/10.14422/PEN.V72.I273.Y2016.002

Corona, S. y Rodríguez, Z. (2000). El amor como vínculo social, discurso e historia: aproximaciones bibliográficas. Espiral, Estudios sobre Estado y Sociedad, VI(17), 49-70.

De la Hermosa, M. (2018). ¿Es la psicoterapia amatonormativa? Amor, afectos y psicoterapia. ¿qué podemos aprender de las relaciones fuera de la norma? En Climent, M. y Carmona, M. (Coords.), Transpsiquiatría. Abordajes queer en salud mental (pp. 222-248). Asociación Española de Neuropsiquiatría.

De Olivera, P., Martins- Silva, Z., Araujo, T. y Da Silva, A. (2013). Teorias sobre o amor no campo de psicologia social. Psicologia, Ciência e Profissão, 33(1), 16-31.

Espinosa, A., Correa, F., García y Barragán, L. (2014) Percepción social de la infidelidad y estilos de amor en la pareja. Enseñanza e Investigación en Psicología, 19(1), 135-147.

Falavigna, G. (2008). La infidelidad como constructo social. ¿Una cuestión moral o una cuestión cultural? En Cabray, M. (Comp.), Entre las transformaciones socioculturales $y$ las construcciones subjetivas: adolescencias $y$ juventudes en transición (pp. 69-82). Brujas.
Ferrer, V., Bosch, E., Navarro, C., Ramis, M.C. y García, E. (2008). El concepto de amor en España. Psicothema, 20(4), 589-595.

Fromm, E. (1959/2015). El arte de amar. Paidós.

García, F., Fuentes, R. y Sánchez, A. (2016). Amor, satisfacción en la pareja y resolución de conflictos en adultos jóvenes. Ajayu, 14(2), 284-302.

García Villanueva, J., Hernández Ramírez, C. y Monter Arizmendi, N. (2019). Amor romántico entre estudiantes universitarios (hombres y mujeres, una mirada desde la perspectiva de género. Revista de Estudios de Género La Ventana, 49, 218-247.

García, M., Salvador, A. y Guzmán, R. (2012). Actitudes hacia la transformación de la vida en pareja: soltería, matrimonio y unión libre. Psicología Iberoamericana, 20(2), 16-25

Gonzáles, J., Martínez-Taboas, A. y Martínez, D. (2009). Factores psicológicos asociados a la infidelidad sexual y/o emocional y su relación a la búsqueda de sensaciones en parejas puertorriqueñas. Revista Puertorriqueña de Psicología, 20, 59-81.

Giddens, A. (2010). La transformación de la intimidad: sexualidad, amor y erotismo en las sociedades modernas. Cátedra.

Hernández, A., González, J. y Regino, D. (2016). Análisis comparado por género del significado de amor de pareja en jóvenes y adultos/as. Revista Electrónica de Psicología Iztacala, 19(4), 1488-1504.

Herrera, M. (2016, abril). Amor romántico $y$ monogamia: una conjunción acrítica $y$ peligrosa. Presentado en IV Jornadas del Centro Interdisciplinario de Investigaciones en Género de la Facultad de Humanidades y Ciencias de la Educación UNLP, La Plata.

IBM Corp. (2017). IBM SPSS Statistics for Windows (Version 25.0). [software]. IBM Corp.

Jodelet, D. (1986). La representación social: fenómeno, concepto y teoría. En Moscovici, S. (Ed.) Psicología social II (pp. 469-494). Paidós

Jisu, H., Delorme, D. E., y Reid, L. N. (2006). Perceived third-person effects and consumer attitudes on prevetting and banning DTC advertising. Journal of Consumer Affairs, 40(1), 90-116.

Instituto Ecuatoriano de Estadísticas y Censos (2017). Los divorcios crecieron $83.45 \%$ en diez años en Ecuador. Recuperado de: HTTP://Www. ECUADORENCIFRAS.GOB.EC/LOS-DIVORCIOSCRECIERON-8345-EN-DIEZ-ANOS-EN-ECUADOR/. Fecha de consulta: 22/05/2019. 
Ley Orgánica para la Prevención y Erradicación de la Violencia de Género contra las Mujeres (2018). Recuperado de: HTtP://WWw.CORDICOM.Gob. EC/WP-CONTENT/UPLOADS/DOWNLOADS/2018/06/ LEY \% 2 O PA R A \% 2 O P R E V E I R \% 2 OY \% 20 ERRADICAR\%2OLA\%2OVIOLENCIA\%2OCONTRA\%20 LA\%2OMUJER.PDF. Fecha de consulta: 22/05/2019.

Maureira, F. (2011a). Los cuatro componentes de la relación de pareja. Revista Electrónica de Psicología Iztacala, 14(1), 321-332.

Maureira, F. (2011b). Neurobiología del amor romántico y la fidelidad. Psiquiatría Universitaria, 7(1), 59-68.

Maureira, F., Flores, E., Rojas, J., Montiel, A., Valenzuela, R. y Veganzones, P. (2017). Autopercepción del compromiso, intimidad, romance y amor en una muestra chilena. Revista Electrónica de Psicología Iztacala, 20(3), 1046-1056.

Montenegro, S. (2013). Coeducación: del ideal del amor romántico a la violencia de género. Tesis de grado, Universidad de la Rioja.

Moscovici, S. (1988). Notes towards a description of social representations. European Journal of Social Psychology, 18, 211-250.

Nuñez, F., Cantó-Milà, N. y Seebach, S. (2015) Confianza, mentira y traición: el papel de la confianza y sus sombras en las relaciones de pareja. Sociológica, 30(84), 117-142.

Ojeda, A., Torres, T. y Moreira, M. (2010). ¿Amor y compromiso en la pareja?: dela teoría a la práctica. Revista Iberoamericana de Diagnóstico y Evaluación, 2(30), 125-142.

Perez, T. y Palma, Y. (2018). Amar amores: o poliamor na contemporaneidade. Psicologia y Sociedade, 30, 1-11. HTTP://DX.DOI.ORG/10.1590/18070310/2018v30165759.

Pinto, B. (2012). Psicología del amor. Departamento de Psicología de la Universidad Católica Boliviana "San Pablo".

Rihm, A., Sharim, D., Barrietos, J., Araya, C. y Larraín, M. (2017). Experiencias subjetivas de intimidad en pareja: un dilema social contemporáneo. Psykhe, 26(2), 1-14. нттр:// DX.DOI.ORG/10.7764/PSYKHE.26.2.1017

Rocha, B., Avendaño, C., Barrios, M. y Polo, A. (2016). Actitudes hacia el amor en relaciones románticas de jóvenes universitarios. Praxis y Saber, 8(16), 155-178. HTTP://DX.DOI.ORG/10.19053/22160159. V7.N15.2016.5727

Rodríguez Salazar, T. (2013). Convergencias conceptuales entre las representaciones sociales y los modelos culturales. Revista CES Psicología, 6(1), 77-103.
Rodríguez Salazar, T., \& Pérez Daniel, R. (2007). Representaciones sociales del amor en jóvenes urbanos. En Luna, R. \& Scribano, A.(Comps.). Contigo aprendí. Estudios sociales sobre las emociones. Centro de Estudios AvanzadosUniversidad Nacional de Córdoba, 173-217.

Rodríguez-Santero, J, García-Carpintero, $\mathrm{M}$. y Porcel, A. (2017). Los estilos de amor en estudiantes universitarios. Diferencias en función del sexo-género. Revista Internacional de Sociología, 75(3), 1-13. HTTP://DX.DOI. ORG/10.3989/RIS.2017.75·3.15.171

Sánchez-Sicilia, A. y Cubells, J. (2018). Amor, posmodernidad y perspectiva de género: entre el amor romántico y el amor líquido. Investigaciones Feministas, 9(1), 151-171. HTTP://DX.DOI. ORG/10.5209/INFE. 58143

Seelig, B. y Rosof, L. (2001). Normal and pathological altruism. Journal of the American Psychoanalytic Association, 49(3), 933-959.

Sirvent, C. (2011). Fidelidad y compromiso en la relación de pareja (el trinomio fidelidad, compromiso y monogamia). Norte de Salud Mental, IX(40), 57-71.

Steger, D., Schroeders, U. y Gnambs, T. (2020). A meta-analysis of test scores in proctored and unproctored ability assessments. European Journal of Psychological Assessment, 36(1), 174184. HTTPS://DOI.ORG/10.1027/1015-5759/A000494

Sternberg, R. (1986). A triangular theory of love. Psychological Review, 93(2), 119-135.

Tendencias Digitales (2010). Hábitos sexuales del latinoamericano. Recuperado de: HTTP:// WWW.UNIFERTES.COM/FILEADMIN/USER_ UPLOAD/IMAGES/HABITOS_SEXUALES_DE_ LATINOAMERICANOS.PDF. FECHA DE CONSULTA: 22/05/2019.

Torres, T. y Ojeda, A. (2009). El compromiso y la estabilidad en pareja: definición y dimensiones dentro de la población mexicana. Psicología Iberoamericana, 17(1), 38-47.

Vaske, J. J., Beaman, J., y Sponarski, C. C. (2017). Rethinking internal consistency in Cronbach's alpha. Leisure Sciences, 39(2), 163-173. HтTPs:// DOI.ORG/10.1080/01490400.2015.1127189

Yela, C. (2012). Análisis de la teoría de las estrategias sexuales en población española. Psicothema, 24 (1), 48-54. 